\title{
Contact With Myelin Evokes a Release of Calcium From Internal Stores in Neonatal Rat Oligodendrocytes In Vitro
}

\author{
STEPHEN J. MOORMAN AND RICHARD I. HUME \\ Department of Biology, University of Michigan, Ann Arbor, Michigan 48109
}

\begin{abstract}
KEY WORDS FURA2, Leading-edge morphology
\end{abstract}
\begin{abstract}
The response of neonatal rat oligodendrocytes to contact with myelin extracts prepared from the central and peripheral nervous system was examined. Contact with either CNS myelin or PNS myelin resulted in collapse of the fine structure of the leading edge of oligodendrocytes in vitro. The collapse of the fine structure of oligodendrocyte processes was preceded by a substantial (approximately fivefold) increase in intracellular free calcium concentration. The calcium concentration increase was due, at least in part, to a release of calcium from internal stores, since it persisted when extracellular calcium was removed by chelation with EGTA. The increase in calcium concentration and the coincident morphological change suggest that oligodendrocytes might be able to recognize and react to specific molecules on the surface of other oligodendrocytes. (c) 1994 Wiley-Liss, Inc.
\end{abstract}

\section{INTRODUCTION}

In the vertebrate central nervous system, the cellular interactions by which the myelin sheath is constructed by oligodendrocytes involve extensive movements by the oligodendrocyte (Raine, 1984). In vitro, oligodendrocytes are highly motile cells (Raff et al., 1978) that actively extend processes that resemble neurites and end in growth cone-like expansions (Fok-Seang and Miller, 1992; Wood and Bunge, 1984; see also Fig. 1). By convention, the distal end of an oligodendrocyte process is referred to as the leading edge, rather than as the growth cone. It is well established that neuronal growth cones are responsible for detecting and integrating environmental cues that affect the development of axons; by analogy, the leading edge of an oligodendrocyte might serve a similar role.

Molecules that can influence growth cone elongation have been identified on neuronal surfaces (Baier and Bonhoeffer, 1992), in the extracellular space (Davies et al., 1990), and on the surface of non-neuronal cells (Caroni and Schwab, 1988a,b). In the intact brain, oligodendrocytes are in close proximity to axons, to astrocytes, and to other oligodendrocytes. Unquestionably, interactions between oligodendrocytes and axons play a critical role in the process of myelination. However, interactions between adjacent oligodendrocytes might also play a role. As an initial approach to determining whether oligodendrocytes might bear surface molecules that influence the development of adjacent oligodendrocytes, we determined the reaction of neonatal rat oligodendrocytes in culture to an application of crude myelin extract. The advantage of this approach is that it is possible to test many different oligodendrocytespecific molecules simultaneously.

\section{MATERIALS ANIJ METHODS}

Preparation and Identification of Oligodendrocytes

Neonatal Sprague-Dawley rats, 1-5 days old, were euthanized by decapitation. The skin and calvaria were removed using fine dissection scissors. The brain and brainstem were removed intact and placed in Puck's

\footnotetext{
Received November 23, 1992; accepted September 27, 1993.
}

Address reprint requests to Stephen J. Moorman, Dept. of Anatomy and Cell Biology, UNT Health Science Center at Fort Worth, 3500 Camp Bowie Blvd., Fort Worth, TX 76107-2699. 

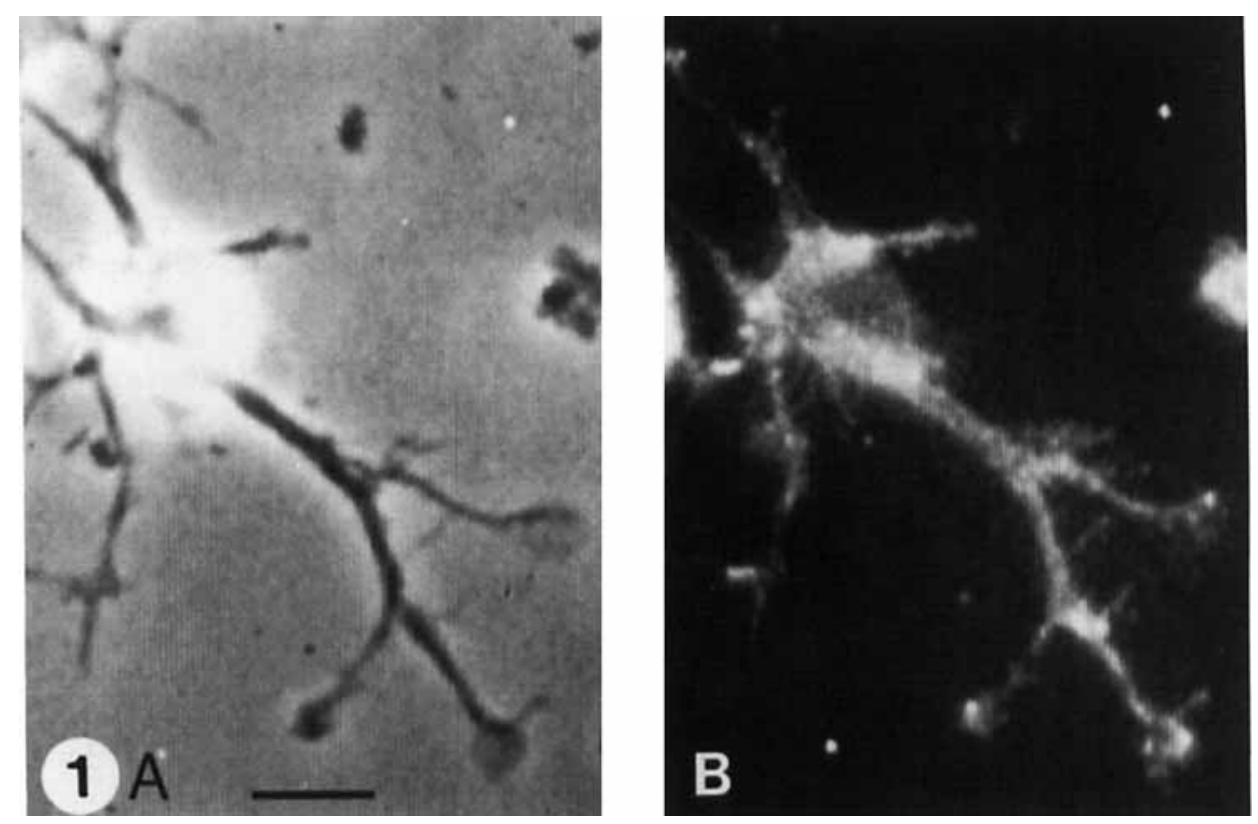

Fig. 1. Identification of oligodendrocytes cultured for 5 days. A: Phase contrast video-microscopy image of a cell identified as an oligodendrocyte based on morphology. B: Fluorescence video-microscopy image of the same cell stained with an antibody to galactocerebroside and a rhodamine-conjugated secondary antibody. This oligodendrocyte had a morphology typical of those used in our experiments. Scale bar = $10 \mu \mathrm{M}$.

solution. All solutions and media had glucose added to bring the total concentration to $6 \% \mathrm{wt} / \mathrm{vol}$. The brainstem was transected both rostral and caudal to the cerebellum to yield a slice of tissue that included the region of brainstem immediately ventral to the cerebellum, the entire cerebellum, and cerebellar peduncles. A small piece of brainstem was then removed from the lateral portion of the floor of the fourth ventricle adjacent to the cerebellar peduncles on both sides. These two pieces of brainstem were placed in a $0.01 \%$ trypsin in Puck's solution for $24 \mathrm{~min}$ at $36^{\circ} \mathrm{C}$. After enzyme treatment, the tissue was triturated and spun twice at 1,000 RPM through $4 \%$ bovine serum albumin (BSA) in media, to remove cellular debris. Dissociated cells were plated in modified tissue culture dishes (Honig and Hume, 1986) coated with poly-d-lysine as a substratum (Letourneau, 1975). Cells were grown in F-12 media (Gibco) containing added glucose $(4.2 \%), 8 \mu \mathrm{g} / \mathrm{ml}$ insulin, $40 \mu \mathrm{g} / \mathrm{ml}$ conalbumin, $12 \mu \mathrm{g} / \mathrm{ml}$ ascorbic acid, $2 \%$ heat inactivated horse serum, $50 \mathrm{ng} / \mathrm{ml}$ 7S-nerve growth factor (NGF), $50 \mathrm{U} / \mathrm{ml}$ penicillin, $0.1 \%$ chick embryo extract, and $0.1 \%$ neonatal rat brain extract.

Neonatal rat brain extract was prepared in the following manner. Brains were removed from 15 rat pups at 5 days of age and placed in $50 \mathrm{ml}$ of Puck's balanced salt solution. The tissue was homogenized and stored at $-70^{\circ} \mathrm{C}$ overnight. The tissue was thawed, re-homogenized, and re-frozen at $-70^{\circ} \mathrm{C}$ overnight. The homogenate was centrifuged at $3,000 \mathrm{RPM}$ for $30 \mathrm{~min}$. The supernatant was removed and re-homogenized. That homogenate was then centrifuged at 3,000 RPM for $30 \mathrm{~min}$. The supernatant was removed and filtered $(0.22$ $\mu \mathrm{m})$.

Under these conditions, the cultures contained a mixture of neurons and glia. Cells were identified by their characteristic morphology when viewed with phase contrast microscopy (Wood and Bunge, 1984). The accuracy of visual identification was verified with immunocytochemical staining. Cells classified as neurons had round, phase bright cell bodies with processes that, after 5-7 days in culture, were relatively long. Cells classified as astrocytes had flat cell bodies. In eight cultures stained with an antibody against glial fibrillary acidic protein (Boehringer Mannheim), $>95 \%$ of the cells classified as astrocytes based on phase contrast microscopy were immunoreactive. Cells classified as oligodendrocytes had small, round, phase bright cell bodies, an eccentric nucleus, and a few short thick processes. In four cultures stained with an antibody against galactocerebroside (Boehringer Mannheim), an oligodendrocyte, and Schwann cell-specific glycolipid (Raff et al., 1978) (Fig. 1), $>85 \%$ of the cells classified as oligodendrocytes based on phase contrast microscopy were immunoreactive.

\section{Preparation of Myelin}

Extracts of CNS myelin were prepared from adult rat spinal cords. Extracts of PNS myelin were prepared 
from adult rat ischiatic nerve. Both extracts were prepared in the following manner (Norton, 1976):

Step 1: Crude fraction isolation: $4.5-6.0 \mathrm{~g}$ of adult nervous tissue (spinal cord or ischiatic nerve) was homogenized in $50 \mathrm{ml}$ ice-cold $0.35 \mathrm{M}$ sucrose. After homogenization, the volume was adjusted to $100 \mathrm{ml}$ with $0.35 \mathrm{M}$ sucrose. Homogenate $(25 \mathrm{ml})$ was layered over $25 \mathrm{ml}$ of $0.85 \mathrm{M}$ sucrose in each of four centrifuge tubes and centrifuged at $48,000 \mathrm{~g}$ for $30 \mathrm{~min}$. The layer of crude myelin that formed at the interface of the two sucrose solutions was collected with a Pasteur pipette.

Step 2: Sucrose washout: the combined myelin layers were suspended in $10 \mathrm{mM}$ HEPES (pH 7.2) in water by homogenization and brought to a final volume of 180 $\mathrm{ml}$. This suspension was centrifuged at $48,000 \mathrm{~g}$ for 15 min and the supernatant discarded.

Step 3: Osmotic shock: the myelin pellets were again dispersed in $180 \mathrm{ml}$ of $10 \mathrm{mM}$ HEPES in water and centrifuged at $12,000 \mathrm{~g}$ for $10 \mathrm{~min}$. The supernatant was discarded. This step was repeated twice.

Step 4: Purified myelin: the myelin pellet was suspended in $0.35 \mathrm{M}$ sucrose, layered over $0.85 \mathrm{M}$ sucrose in each of four tubes, and centrifuged as in step 1 . The purified myelin was removed from the interface using a pasture pipette.

Step 2 was repeated at the end to yield a pellet of myelin in $10 \mathrm{mM}$ HEPES ( $\mathrm{pH} 7.2)$ in water. This pellet was aliquoted into $50 \mu \mathrm{l}$ samples and stored at $-70^{\circ} \mathrm{C}$ until used. Immediately prior to use, $75 \mu \mathrm{l}$ of media was added to an aliquot of myelin. The osmolality of the media added to the myelin extract was adjusted with sucrose so that when added to the myelin the final osmolality was $300 \mathrm{mOs}$. Myelin extract was placed in contact with oligodendrocytes by extrusion from a puffer pipette.

In order to test whether the effect evoked by myelin was specific to an extract of nervous tissue, we tried preparing separate extracts of adult rat liver and adult rat kidneys using the same protocol that was used to make the myelin extracts. However, no material was present in the final pellet.

For certain experiments, the CNS myelin extract was heat-inactivated by maintaining the extract at $100^{\circ} \mathrm{C}$ for $30 \mathrm{~min}$. For other experiments, the CNS myelin extract was treated with $0.02 \%$ trypsin at $37^{\circ} \mathrm{C}$ for 30 min.

\section{Video-Microscopy}

We made video microscopic observations of oligodendrocytes using an inverted microscope that was modified so that the stage, objectives, and culture dish were maintained at $37^{\circ} \mathrm{C}$. Before the culture dish was placed on the microscope, the normal media was replaced by media containing $20 \mathrm{mM}$ HEPES ( $\mathrm{pH} 7.3$ ), and then preheated mineral oil was placed over the media to prevent evaporation. Using mineral oil allowed access to the cells for myelin application without degradation of either the fluorescent or phase contrast images. Cells were initially identified using phase contrast optics. The total apparent surface area of the leading edge was measured by tracing the outline of the leading edge and calculating the area within the outline. If the apparent surface area decreased by more than $50 \%$, the leading edge was scored as having collapsed.

\section{FURA2 Microscopy}

Cells were loaded with FURA-2, AM (Molecular Probes) by incubating them in a solution containing: $\mathrm{CaCl}_{2}, 5$ $\mathrm{mM}$; TEA-Cl, $120 \mathrm{mM}$; $\mathrm{MgCl}_{2}, 2 \mathrm{mM}$; HEPES, $10 \mathrm{mM}$; FURA-2, AM, $3 \mu \mathrm{M}$; and Pluronic detergent, $1 \% \mathrm{wt} / \mathrm{vol}$. The $\mathrm{pH}$ was adjusted to 7.3 with $\mathrm{CsOH}$. The osmolality was adjusted to $300 \mathrm{mOs}$ with sucrose. Cells were incubated in the FURA2 solution for 90 min at $37^{\circ} \mathrm{C}$, rinsed twice with normal media, and returned to the incubator for $60 \mathrm{~min}$ before being placed on the microscope stage for experimental observation.

FURA2 ratio measurements were made using two different systems: a photometry system (Delta Scan, Photon Technology Inc.) attached to a Nikon DiaPhot microscope with a $40 \times$ FLUOR oil-immersion objective, and a video-based system (Image 1/FL, Universal Imaging Inc.) that used an SIT camera mounted on a Zeiss IM inverted microscope equipped with a computer-controlled shutter and filter wheel and a $40 \times-U V$ glycerine-immersion objective. For the photometry system, the excitation was provided by a xenon bulb. The exciting light was passed through dual monochrometers centered at 350 and $380 \mathrm{~nm}$. For the video system, the excitation was provided by a mercury bulb. The fluorescence in response to excitation through $10 \mathrm{~nm}$-wide band pass filters centered at 350 and $380 \mathrm{~nm}$ was measured. Ratio measurements were made 20 times/s on the photometry system, but images could be acquired and saved only once very $3-4 \mathrm{~s}$ on the video system. The photometry system allowed data to be collected more rapidly, at the loss of spatial resolution. The photometry system was equipped with adjustable shutters in the light path between the specimen and the detector. This allowed emission intensity to be measured for one rectangular area of interest in the field of view. In the experiments using the photometry system, only the emission intensity for the leading edge was measured. All experiments using the photometry system were performed at room temperature, and all experiments using the video system were performed at $37^{\circ} \mathrm{C}$.

For each oligodendrocyte studied, an initial series of measurements were made to verify that there was a steady baseline calcium concentration. The final $25 \mathrm{~s}$ of the baseline data were stored, and then the acquisition program was paused. A myelin-containing puffer pipette was then brought close to the oligodendrocyte. Myelin was puffed onto the oligodendrocyte, and the micropipette was removed from the dish. Data acquisition was then resumed. This procedure, which took 15-30 $\mathrm{s}$, was necessary because the pipette degraded the im- 
age when it was in the field. The ratios of the background-corrected amplitude of fluorescence values were converted into free calcium concentrations using two methods. A calibration curve of ratio values vs. calcium concentration was generated using FURA2-free acid in solutions of known free calcium concentrations. Ratio values were also converted to calcium concentrations using the formula of Grynkiewicz et al. (1985). For the photometry system $F_{\min }$ (the signal intensity in response to $380 \mathrm{~nm}$ excitation with 0 calcium) and $F_{\max }$ (the signal in response to $380 \mathrm{~nm}$ excitation with very high calcium) were $1.174 \times 106$ and $8.416 \times 104$, respectively (in units of photons counted), and the maximal and minimal fluorescence ratios $\left(R_{\min }\right.$ and $\left.R_{\max }\right)$ were 0.48 and 22.7, respectively. For the video system $F_{\min }$ and $F_{\max }$ (in $A D$ converter units) were 151.34 and 10.81, respectively, while $R_{\min }$ and $R_{\max }$ were 0.28 and 5.76 , respectively. For both systems a Kd of $224 \mu \mathrm{M}$ was used. The two calibration methods yielded calcium concentrations that were within $10 \%$ of each other. All drugs tested were made as concentrated stocks and then diluted into both the medium bathing the cells and into the myelin-containing solution in the puffer pipette. A t-test for means from unequal sample sizes was used to compare average resting internal free calcium concentration $\left([\mathrm{Ca}]_{i}\right)$ levels in each group, average maximal $[\mathrm{Ca}]_{i}$ levels reached after contact with myelin in each group, and average change in $[\mathrm{Ca}]_{\mathrm{i}}$ evoked by contact with myelin in each group.

For certain experiments, the surface area of leading edges of oligodendrocytes was monitored using fluorescence video-microscopy. This approach was taken because the myelin extract was highly refractive, and did not allow for high resolution observation of leading edges with phase contrast or Nomarski optics during the time that the myelin was in contact with the leading edge. Once every $5 \mathrm{~min}$, the FURA2-loaded oligodendrocyte was excited using only $380 \mathrm{~nm}$ light. Eight fluorescence video images were acquired, averaged, and saved to disk. At the end of each experiment, the surface area of the leading edge was measured on each of the saved images. Using the Image-1 software, the leading edge's outline was traced and the area within the outline calculated. When excited with $380 \mathrm{~nm}$ light, the intensity of emission of FURA2 decreases with increasing calcium concentration. However, even at the highest concentration of calcium studied, the fluorescence when excited at $380 \mathrm{~nm}$ was quite bright, and the entire outline of the leading edge could easily be distinguished. We confirmed that measurements of the surface area of the leading edge from the fluorescence in response to $380 \mathrm{~nm}$ excitation gave an accurate estimate in the following manner. At the end of each experiment, the myelin extract was washed off and a phase contrast image and a fluorescent image of each leading edge was acquired. The surface areas obtained from the two images were measured and compared. Measurements from the two types of video-images were not significantly different $(P<0.01)$. A final concern might be that when calcium is elevated, the decrease in fluorescence inten- sity might lead to an underestimate of surface area. The experiments in which calcium was elevated by applying A23187 indicate that this is unlikely as well. There was no discrepancy in surface area estimated from phase and fluorescence in response to $380 \mathrm{~nm}$ excitation when calcium was caused to rise to levels even higher than that elicited by myelin. This result is not at all surprising, since the original work of Grynkiewicz et al. (1985) showed that the fractional decrease in fluorescence over this range of calcium concentrations is not very large.

\section{RESULTS \\ Leading Edge Morphology of Rat Oligodendrocy tes Changed on Contact With Myelin}

Video microscopic observations were made from neonatal rat oligodendrocytes in culture for a period of 5-7 days. No more than three cells were studied in a single dish, and all results reported include cells obtained from several different platings. In total, over 100 different dishes of cells were used for these studies. The stage of the microscope was maintained at $37^{\circ} \mathrm{C}$. Our major results are shown photographically in Figures 2 and 3 and graphically in Figure 4. In the absence of contact with other cells, the processes of oligodendrocytes extended and retracted filopodia and lamellapodia, but changed little in overall complexity over a $30 \mathrm{~min}$ time period (Fig. 2). Continuous observation of control oligodendrocytes by phase contrast microscopy for $30 \mathrm{~min}$ $(\mathrm{n}=7)$ indicated that, on average, there was a slight increase in the surface area of the leading edge (Fig. 4). In contrast, when myelin was applied for $30 \mathrm{~min}$ and then washed off so that the leading edge could be visualized with phase contrast microscopy $(n=25)$, a dramatic change in morphology was found. The fine filopodia and lamellapodia at the leading edge were retracted, so that the overall appearance of the process was much smaller and smoother. In the example illustrated two of the fine processes retracted completely into the cell body (Fig. 2) within $30 \mathrm{~min}$. To quantify this change in morphology, we measured the total apparent surface area (not including any filopodia) of the leading edge. The leading edge was scored as having collapsed if the surface area decreased by $50 \%$ or more. By these criteria, 23 of 25 processes collapsed within 30 min when contacted with CNS myelin extract.

As a control for any nonspecific contact-mediated effects evoking collapse of the fine structure of the leading edge, we heated the extract of CNS myelin to $100^{\circ} \mathrm{C}$ for $30 \mathrm{~min}$ before applying it to oligodendrocytes. The CNS myelin extract treated in this manner did not evoke any change in morphology of the leading edge of oligodendrocytes $(n=10)$. As an additional test for nonspecific effects, we also treated the CNS myelin with trypsin. Trypsin-treated myelin also had no effect on the morphology of the leading edge of oligodendrocytes $(n=10)$.

Because of the refractile nature of the myelin extract, it was not possible to observe the leading edge using 

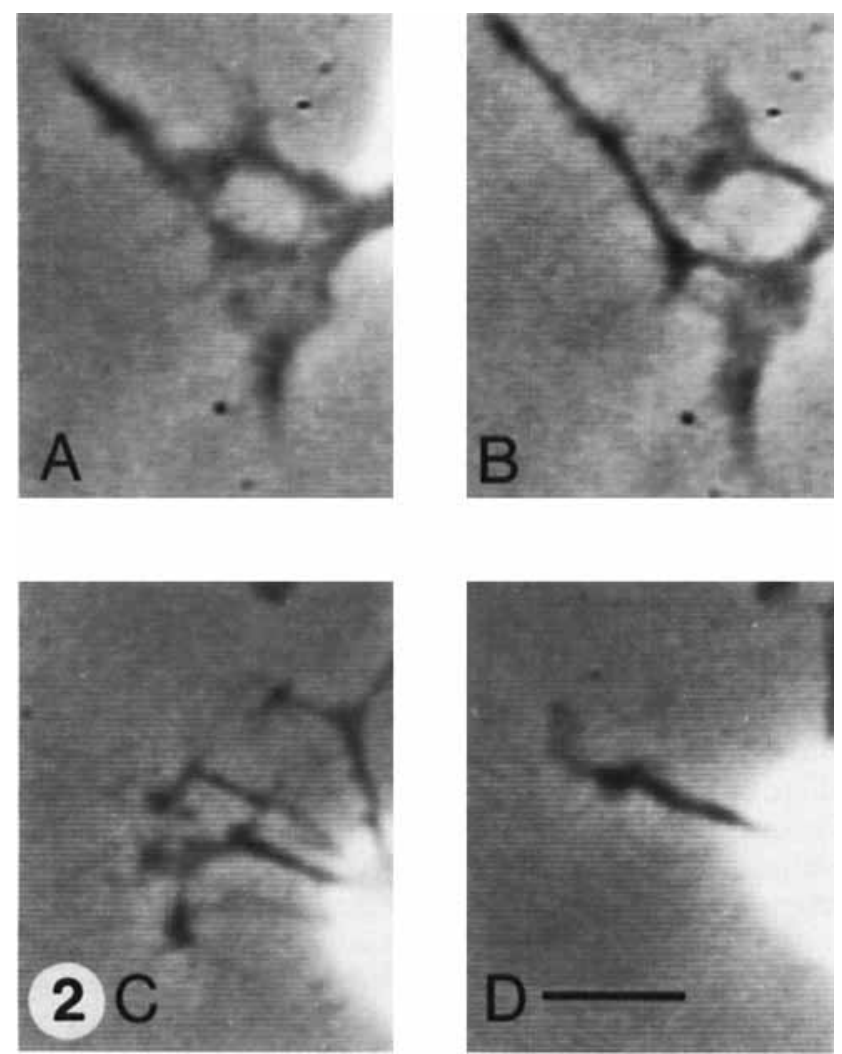

Fig. 2. Time lapse video-microscopy observations of the leading edges of oligodendrocytes at $37^{\circ} \mathrm{C}$. A: Cell that received no treatment. $B$ : Same cell as in A 30 min later. $C$ : Cell that was contacted with CNS myelin extract just after the image was collected. D: At the end of a 30 min period the myelin was removed. Scale bar $=10 \mu \mathrm{M}$.

phase contrast video-microscopy while the leading edge was in contact with the myelin extract. Therefore, to determine the time course of the collapse of the leading edge, we monitored the morphology of the leading edge every $5 \mathrm{~min}$ using fluorescence microscopy to observe cells loaded with FURA2 (Figs. 3, 4). We first monitored several $(n=3)$ leading edges using this technique without applying any myelin extract. Under this condition, the surface area of the leading edges increased slightly over a $30 \mathrm{~min}$ period, similar to the leading edges observed with phase contrast video-microscopy (Fig. 4). Thus, brief fluorescence illumination did not damage the leading edge. When the surface area of oligodendrocyte leading edges in contact with myelin was assessed by fluorescent microscopy $(n=8$; Fig. 4$)$, a substantial decrease in surface area was seen within $5 \mathrm{~min}$. Within 10 min after application of the CNS myelin extract, the leading edge had decreased in surface area by more than $60 \%$, and a slight additional decrease in surface area was seen in the next 20 min (Fig. 4).

\section{CNS Myelin Evoked an Increase in Intracellular Free Calcium}

Changes in $[\mathrm{Ca}]_{\mathrm{i}}$ have been associated with many different growth cone behaviors including growth cone collapse (Kater and Mills, 1991). If the analogy to the neuronal growth cone holds, then changes in $[\mathrm{Ca}]_{i}$ in the oligodendrocyte leading edge might accompany recognition of and response to environmental signals.

Application of CNS myelin extract to oligodendrocytes consistently caused a large increase in $[\mathrm{Ca}]_{\mathrm{i}}$. In the majority of experiments, the intracellular free calcium concentration $\left([\mathrm{Ca}]_{\mathrm{i}}\right)$ within the distal processes and leading edges of neonatal rat oligodendrocytes loaded with FURA2 was measured with a photometry system. In these experiments, cells were bathed in our standard media, and maintained at room temperature. On average the resting intracellular free calcium increased by more than a factor of 5 , from $71 \mathrm{nM}$ to $431 \mathrm{nM}$ (Table 1 ). Similar results were obtained when the experiments were performed using the video system with the cells at $37^{\circ} \mathrm{C}$ (average maximum $[\mathrm{Ca}]_{\mathrm{i}}=379 \pm 13, \mathrm{n}=10$ ).

The increase in $[\mathrm{Ca}]_{\mathrm{i}}$ occurred rapidly, even at room temperature (Fig. 5). In more than half the cells (41 of 70), the increase reached a peak during the 20-30 s that the data acquisition program was paused. Typically, the $[\mathrm{Ca}]_{\mathrm{i}}$ returned to pre-application levels with a time course that was much slower than the increase (1.5-2 $\min )$, and occasionally $(n=6)$, the $[\mathrm{Ca}]_{i}$ increased a second time during the 6 min that $[\mathrm{Ca}]_{\mathrm{i}}$ was monitored. This increase in $[\mathrm{Ca}]_{i}$ in response to myelin did not occur if the myelin was pretreated by incubation at $100^{\circ} \mathrm{C}$ or by trypsin treatment (Table 1 ).

\section{Specificity of the Myelin-Induced Responses}

Not all cells of the nervous system respond to myelin extract. Astrocytes $(n=6)$ were non-responsive. Astrocytes had a resting calcium level of $82 \mathrm{~nm}( \pm 8, \mathrm{n}=6)$ before and after application of CNS myelin extract.

Contact with myelin from the peripheral nervous system of the adult rat evoked a $[\mathrm{Ca}]_{i}$ increase in oligodendrocytes in vitro (Fig. 5, Table 1) and caused collapse of the leading edges of eight of ten cells tested. The $[\mathrm{Ca}]_{i}$ increase evoked in oligodendrcicytes by PNS myelin was not significantly different from that evoked by CNS myelin (Table 1).

\section{Role of Elevated [Ca $]_{i}$ in Collapse and Retraction of the Leading Edge}

We tested whether a rise in $[\mathrm{Ca}]_{\mathrm{i}}$ is sufficient to evoke the collapse of the leading edge by using the non-fluorescent calcium ionophore 4-bromo A23187 (Molecular

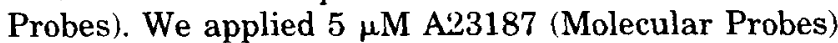
from the same type of puffer pipette that we used for the myelin application and monitored $[\mathrm{Ca}]_{\mathrm{i}}$ using the video-microscopy system. At $37^{\circ} \mathrm{C}$, a $15-20 \mathrm{~s}$ application of A23187 caused $[\mathrm{Ca}]_{\mathrm{i}}$ to rise transiently to $0.8-1.0$ $\mu \mathrm{M}$ and then to return to preapplication levels in $2-3$ min in all oligodendrocyte leading edges tested $(n=10)$. These transients were similar in time course, and slightly larger in magnitude than the calcium transients ob- 

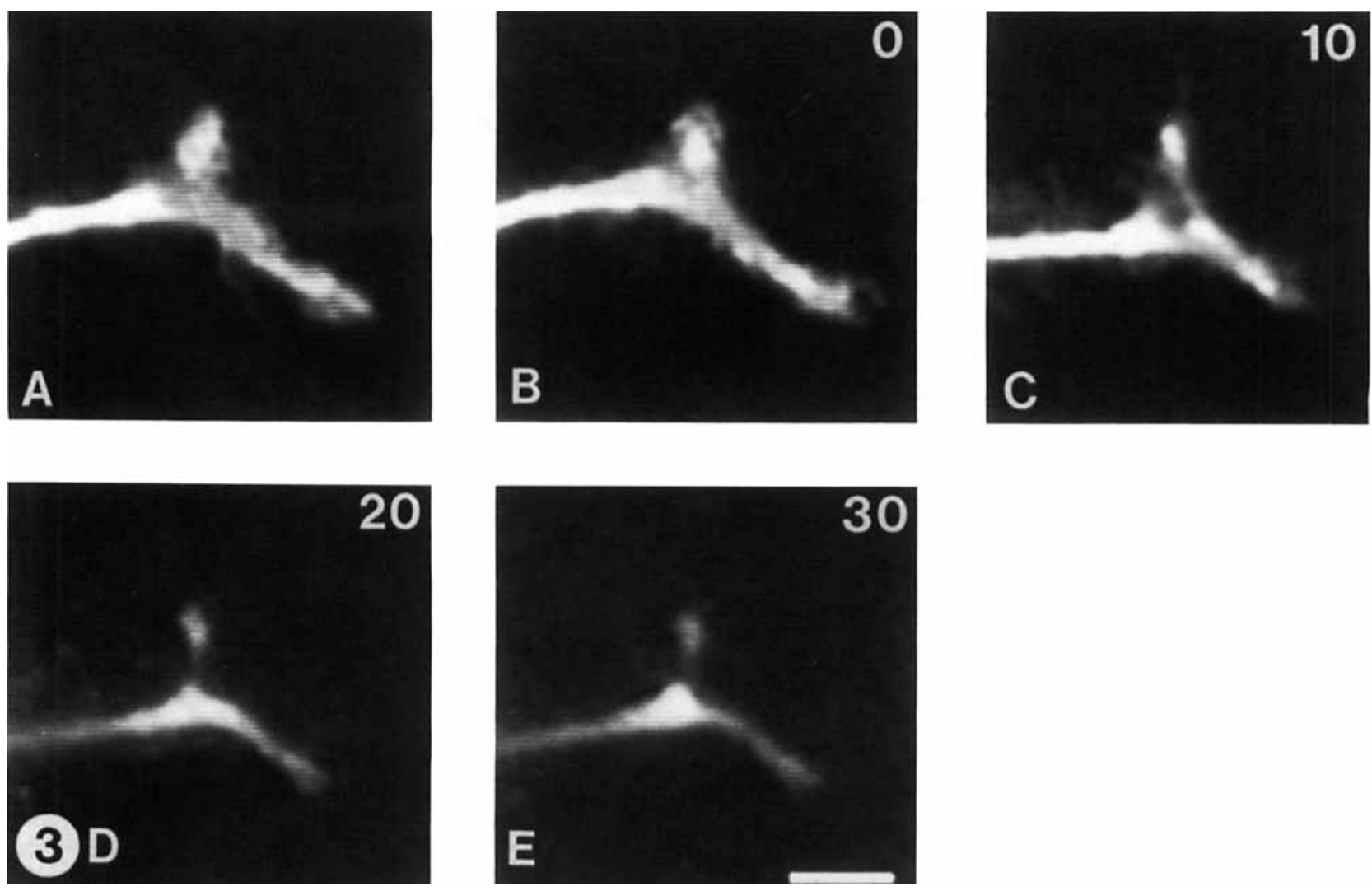

Fig. 3. Collapse of the oligodendrocyte leading edge at $37^{\circ} \mathrm{C}$ in response to contact with CNS myelin extract. The same FURA2-loaded oligodendrocyte leading edge was imaged using fluorescence videomicroscopy, exciting the cell with only $380 \mathrm{nM}$ light, before and after application of CNS-myelin extract. Numbers in the upper right corner represent elapsed time in minutes after image $B$. A: Leading edge immediately before CNS myelin extract was applied. B: The same leading edge immediately after (time $=0$ ) CNS myelin application. C-E: The same leading edge viewed at the times indicated. Scale bar $=10 \mu \mathrm{M}$. served in response to CNS myelin. Under these conditions, the leading edge of seven of ten oligodendrocytes collapsed within $30 \mathrm{~min}$ after application of A23187. The 3 that did not collapse within 30 min were still intact after $60 \mathrm{~min}$.

\section{Myelin Evoked a Release of Calcium From Intracellular Stores and a Transmembrane Influx of Calcium}

A rise in $[\mathrm{Ca}]_{\mathrm{i}}$ might be caused by either a calcium influx, by a release from internal stores, or by a combination of the two. To test a possible release from internal stores, extracellular calcium was chelated, by the addition of $5 \mathrm{mM}$ EGTA. Our calculations indicate that $5 \mathrm{mM}$ EGTA reduces the free calcium concentration in the normal media from $300-400 \mu \mathrm{M}$ to approximately $20-50 \mathrm{nM}$. This free calcium concentration is close to the resting $[\mathrm{Ca}]_{\mathrm{i}}$ we measured in oligodendrocytes, so there would be little or no influx of calcium if calcium channels opened in response to myelin. In this solution, CNS myelin could still evoke a substantial increase in $[\mathrm{Ca}]_{\mathrm{i}}$. However, the peak value reached when cells were

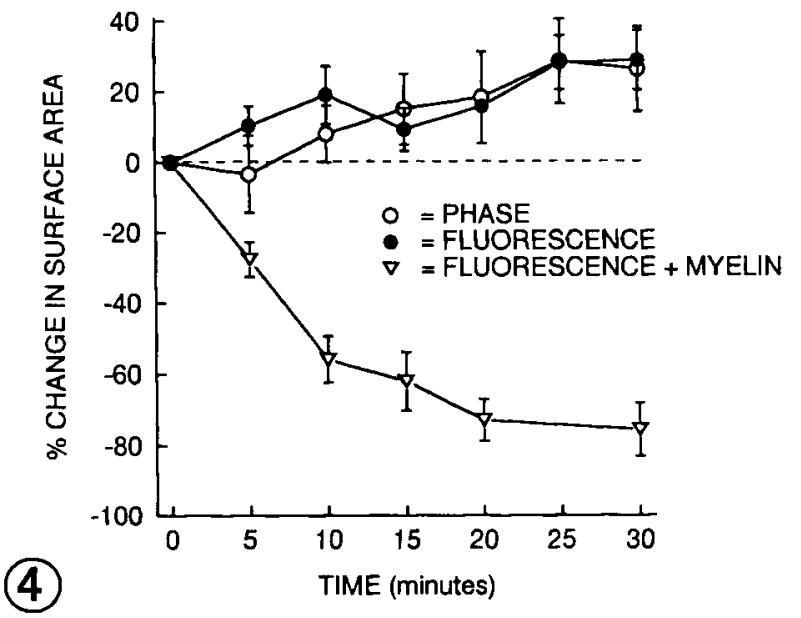

Fig. 4. Percent change in surface area of oligodendrocyte leading edges at $37^{\circ} \mathrm{C}$. Open circles represent oligodendrocyte leading edges $(\mathbf{n}=7)$ that were monitored using phase contrast video-microscopy once every $5 \mathrm{~min}$. Filled circles represent FURA2-loaded oligodendrocyte leading edges $(n=3)$ that were monitored using fluorescence video-microscopy (observation period $=3$ seconds, 3 times $/ \mathrm{min}$ for 30 $\mathrm{min}$ ). Only the data for $5 \mathrm{~min}$ intervals are presented. Open triangles represent FURA2-loaded oligodendrocyte leading edges $(n=8)$ that were monitored using fluorescence video-microscopy once every $5 \mathrm{~min}$ after CNS myelin extract was placed in contact with each leading edge. 


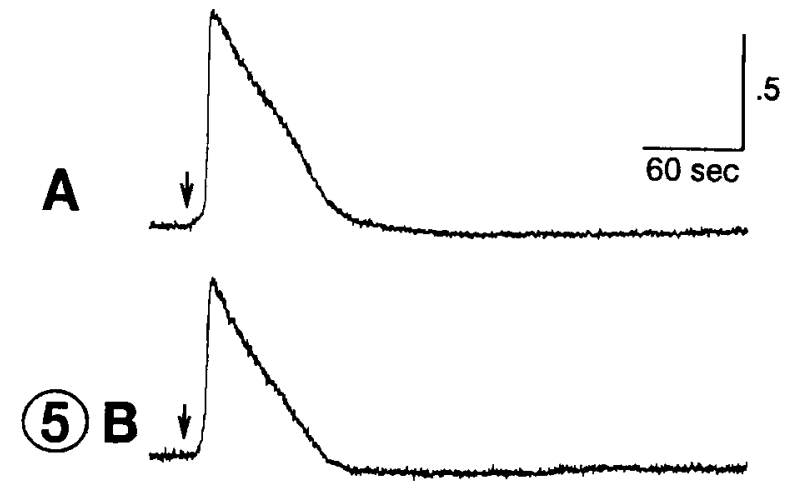

Fig. 5. Calcium concentration changes in the leading edges of oligodendrocytes at room temperature in response to myelin extracts. The arrows indicate the time in each trace at which the myelin extract was applied to the leading edge. Vertical scale indicates the ratio A350/ A380. A: Cell treated with CNS myelin extract. B: Cell treated with PNS myelin extract.

TABLE 1. Effects of myelin extracts on calcium concentration in the leading edge of neonatal rat brainstem oligodendrocytes ${ }^{\text {a }}$

\begin{tabular}{lcc}
\hline Treatment (no.) & Resting $[\mathrm{Ca}]_{\mathbf{i}}$ & {$[\mathrm{Ca}]_{\mathbf{i}}$ maximum } \\
\hline CNS myelin (17) & $71 \pm 2$ & $431 \pm 27$ \\
Heat-inactivated CNS myelin (10) & $73 \pm 3$ & $73 \pm 3$ \\
Trypsin-treated CNS myelin (10) & $71 \pm 3$ & $71 \pm 3$ \\
PNS myelin (10) & $83 \pm 4$ & $437 \pm 33$ \\
CNS myelin & $68 \pm 4$ & $222 \pm 25$ \\
$\quad$ +5 mM EGTA (8) & $70 \pm 5$ & $191 \pm 21$ \\
$\quad+5$ mM Lanthanum (14) & $107 \pm 3$ & $563 \pm 32$ \\
$\quad+50 \mu$ M Nifedipine (19) & $110 \pm 3$ & $522 \pm 38$ \\
$\quad+75 \mu$ M Diphenylhydantoin (9) & $69 \pm 5$ & $493 \pm 10$ \\
\hline
\end{tabular}

${ }^{\mathrm{H}}$ All calcium concentrations are shown in $\mathrm{nM}( \pm \mathrm{SEM})$.

bathed in EGTA was significantly less than in control conditions (Table 1 ). This result indicates that at least a part of the CNS myelin-evoked $[\mathrm{Ca}]_{i}$ increase in oligodendrocytes is due to a release of calcium from internal stores. The partial inhibition of the calcium signal by EGTA might arise because there was also a transmembrane component to the $[\mathrm{Ca}]_{i}$ increase or because there was a partial depletion of calcium stores in the absence of external calcium. To test for a transmembrane component of the myelin-evoked increase in $[\mathrm{Ca}]_{i}$, we added lanthanum to the extracellular solution, since lanthanum is known to block calcium flux through several kinds of channels (Gengo et al., 1983). Lanthanum (5 $\mathrm{mM}$ ) significantly reduced but did not block the myelinevoked increase in $[\mathrm{Ca}]_{\mathrm{i}}$ (Table 1 ). This finding suggests that the myelin-evoked $[\mathrm{Ca}]_{i}$ increase is due to an influx of calcium as well as a release from internal stores.

If a portion of the calcium signal is due to transmembrane calcium flux, it might be possible to block this component with calcium channel blockers. Although it is not known whether mature oligodendrocytes have voltage-gated calcium channels, oligodendrocyte precursors are reported to have T- and L-type voltagegated calcium channels (Verkhratsky et al., 1990). We used diphenylhydantoin in an attempt to block T-type channels (Yaari et al., 1987), and we used nifedipine in an attempt to block L-type channels (Gengo et al., 1983).
Neither diphenylhydantoin nor nifedipine reduced or blocked the $[\mathrm{Ca}]_{i}$ increase (Table 1). In fact, the resting $[\mathrm{Ca}]_{\mathrm{i}}$ levels were slightly higher in the presence of these compounds, and the maximum $[\mathrm{Ca}]_{i}$ levels reached with either drug in the media were significantly higher than control levels (Table 1 ).

$\mathrm{N}$-type calcium channels are thought to be found only in neurons and have been implicated in mediating the calcium influx response for neurotransmitter release at the presynaptic terminal (Pin and Bockaert, 1990; Rivier et al., 1987). Omega-conotoxin GVIA specifically blocks $\mathrm{N}$-type voltage-sensitive calcium channels in neurons (Olivera et al., 1987) and also blocks myelin-induced collapse of locus coeruleus neuron growth cones in vitro (Moorman and Hume, 1993). The change in $[\mathrm{Ca}]_{\mathrm{i}}$ evoked in oligodendrocytes by contact with CNS myelin in media that contained omega-conotoxin GVIA was not significantly different from the $\left[\mathrm{C}_{\mathrm{C}} \mathrm{a}\right]_{\mathrm{i}}$ evoked in the normal media (Table 1). Moreover, omega-conotoxin GVIA did not block collapse of the fine structure of the leading edge of oligodendrocytes in vitro $(n=5)$.

\section{DISCUSSION}

We have shown that an extract of myelin can have profound effects on the behavior of oligodendrocytes in vitro. Since myelin is a product of differentiated oligodendrocytes, these results suggest that oligodendrocytes might be able to recognize and react to specific molecules on the surface of other oligodendrocytes. The significance of these observations to the in vivo behavior of oligodendrocytes is unknown. However, if the collapse of the leading edge of an oligodendrocyte is interpreted as an inhibitory response, as it is for the neuronal growth cone, then one possible role might be to ensure that myelin sheaths are properly spaced, and that adjacent oligodendrocytes do not overgrow the node of Ranvier.

An important issue is whether the change in intracellular free calcium in response to myelin extract is specific to oligodendrocytes. It is clear that oligodendrocytes are not the only cell type that can respond to this extract. Schwab and his colleagues (Bandtlow et al., 1993) have shown that the growth cones of peripheral neurons can respond to CNS myelin extract, and we have obtained similar results in studies of the growth cones of neonatal rat locus coeruleus neurons (Moorman and Hume, 1993). However, not all cells of the nervous system respond to myelin extract since astrocytes were non-responsive.

The experiments described here were performed while doing a parallel set of experiments on the response of brainstem neurons to myelin (Moorman and Hume, 1993). There are interesting parallels and some important differences between the responses of these two cell types. In both brainstem neurons and oligodendrocytes, CNS myelin elicits a collapse of the motile processes. Furthermore, this inhibition of motile activity is accompanied by an increase in $[\mathrm{Ca}]_{i}$. In the case of brainstem neurons, results obtained using an agent that blocks 
the $[\mathrm{Ca}]_{\mathrm{i}}$ increase suggested that the calcium increase was necessary but not sufficient to induce the growth cone collapse (Moorman and Hume, 1993). The results of experiments using the ionophore A23187 on oligodendrocytes are consistent with the idea that calcium might play a role in mediating the collapse of the leading edge. However, since we have been unable to block completely the myelin-induced $[\mathrm{Ca}]_{i}$ increase in oligodendrocytes, a test of calcium's role in mediating the collapse of the leading edge cannot yet be performed.

Interestingly, the mechanism by which calcium concentration is elevated is quite different in brainstem neurons and oligodendrocytes. In brainstem neurons, myelin seems to elevate $[\mathrm{Ca}]_{\mathrm{i}}$ through a mechanism requiring calcium influx through voltage-gated calcium channels (Moorman and Hume, 1993), and a specific calcium channel blocker can block the effect of myelin. In contrast, in oligodendrocytes, release from internal stores seems to be most important, and none of the calcium channel blockers tested, except lanthanum, had any effect. If the calcium rise in oligodendrocytes is in part due to a transmembrane flux (as suggested by the experiments with extracellular lanthanum), the calcium must enter the cells by a different mechanism than in brainstem neurons. Possibilities include a pump, a ligandgated calcium channel or a voltage-gated calcium channel with novel pharmacology. One good candidate mechanism is suggested by the work of Dyer and Benjamins (1990), and involves the molecule galactocerebroside (GalC), a membrane glycoprotein found specifically on oligodendrocytes and Schwann cells. An antibody to GalC has been reported to cause an influx of calcium, and this influx was blocked by extracellular EGTA but not by extracellular cobalt (Dyer and Benjamins, 1990). Furthermore, the calcium influx also led to a rearrangement of cytoskeletal elements that resulted in a collapse of the fine structure of the oligodendrocyte (Dyer and Benjamins, 1990).

The results obtained with peripheral myelin further support the idea that the mechanism by which myelin induces a rise in $[\mathrm{Ca}]_{i}$ differs between neurons and oligodendrocytes. In certain CNS and PNS neurons in vitro, growth cone collapse is evoked by specific components of myelin produced by oligodendrocytes in the rat central nervous system (Caroni and Schwab, 1988a,b; Moorman and Hume, 1993). These components of myelin are not produced by Schwan cells in the rat peripheral nervous system, so myelin from the peripheral nervous system does not evoke growth cone collapse (Caroni and Schwab, 1988a,b; Moorman and Hume, 1993). Moreover, myelin from the rat central nervous system also evokes a $[\mathrm{Ca}]_{i}$ increase in neonatal rat neurons in vitro (Moorman and Hume, 1993; Schmidt et al., 1991), while myelin from the peripheral nervous system does not (Moorman and Hume, 1993). In contrast, peripheral and central myelin were equally effective in inducing morphological changes and an increase in $[\mathrm{Ca}]_{i}$ in oligodendrocytes. This suggests that either there are different molecules in CNS and PNS myelin that can evoke similar effects in oligodendrocytes in vitro, or that a molecule common to both CNS and PNS myelin triggers this response.

\section{ACKNOWLEDGMENTS}

We thank Tim Hassinger for preparing some of the myelin extract. Part of this work was performed while S.J.M. was on a Young Investigator Fellowship at the Marine Biological Laboratory at Woods Hole. The work was supported by N.I.H. grant NS 21043 and a grant from the American Paralysis Association.

\section{REFERENCES}

Baier, H. and Bonhoeffer, F. (1992) Axon guidance by gradients of a target-derived component. Science, 255:472-475.

Bandtlow, C., Schmidt, M.F., Hassinger, T.D., Schwab, M.E., and Kater, S.B. (1993) Role of intracellular calcium in NI-35-evoked collapse of neuronal growth cones. Science, 259:80-83.

Caroni, P. and Schwab, M.E. (1988a) Two membrane protein fractions from rat central myelin with inhibitory properties for neurite growth and fibroblast spreading. J. Cell Biol., 106:1281-1288.

Caroni, P. and Schwab, M.E. (1988b) Antibody against myelin-associated inhibitor of neurite growth neutralizes nonpermissive substrate properties of CNS white matter. Neuron, 1:85-96.

Davies, J.A., Cook, G.M.W., Stern, C.D., and Keynes, R.J. (1990) Isolation from chick somites of a glycoprotein fraction that causes collapse of dorsal root ganglion growth cones. Neuron, 4:11-20.

Dyer, C.A. and Benjamins, J.A. (1990) Glycolipids and transmembrane signaling: Antibodies to galactocerebroside cause an influx of calcium in oligodendrocytes. J. Cell Biol., 111:625-633.

Fok-Seang, J. and Miller, R.H. (1992) Astrocyte precursors in neonatal rat spinal cord cultures. $J$. Neurosci., 12:2751-2764.

Gengo, P.J., Luchowski, E., Rampe, D.E., Rutledge, A., Triggle, A.M. Triggle, D.J., and Janis, R.A. (1983) Chemical and pharmacological approaches to the definition and quantitation of calcium channels. Cold Spring Harbor Symp. Quant. Biol., 48:279-285.

Grynkiewicz, G., Poenie, M., and Tsien, R.Y. (1985) A new generation of calcium indicators with greatly improved fluorescent properties. J. Biol. Chem., 260:2440-3450.

Honig, M.G. and Hume, R.I. (1986) Fluorescent carbocyanine dyes allow living neurons of identified origin to be studied in long-term cultures. J. Cell Biol., 103:171-187.

Kater, S.B. and Mills, L.R. (1991) Regulation of growth cone behavior by calcium. J. Neurosci., 11:891-899.

Letourneau, P.C. (1975) Possible roles for cell-to-substratum adhesion in neuronal morphogenesis. Dev. Biol., 44:77-91

Moorman, S.J. and Hume, R.I. (1993) Omega-conotoxin prevents myelin evoked growth cone collapse in neonatal-rat brainstem neurons in-vitro. J. Neurosci., 13:4727-4736.

Norton, J.S. and Cammer, W. (1984) Isolation and characterization of myelin. In: Myelin. P. Morell, ed. Plenum Press, New York. pp. 147-195.

Norton, W.T. (1976) Isolation of myelin from nerve tissue. In: Methods in Enzymology, Vol. 31. S. Fleischer and L. Packer, eds. Academic Press, New York. pp. 435-444.

Olivera, B.M., Cruz, L.J., de Santos, V., LeCheminant, G.W., Griffin, D., Zeikus, R., McIntosh, J.M., Galyean, R., Varga, J., and Gray, W.R. (1987) Neuronal calcium channel antagonists. Discrimination between calcium channel subtypes using omega-conotoxin from Conus magnus venom. Biochemistry, 26:2086-2090.

Pin, J.P., and Bockaert, J. (1990) Omega-conotoxin GVIA and dihydropyridines discriminate two types of $\mathrm{Ca}^{2+}$ channels involved in GABA release from striatal neurons in culture. Eur. J. Pharmacol., 188: $81-84$

Raff, M.C., Mirsky, R., Fields, K.L., Lisak, R.P., Dorfman, S.H., Silberberg, D.H., Gregson, N.A., Liebowitz, S., and Kennedy, M.C. (1978) Galactocerebroside is a specific cell surface antigenic marker for oligodendrocytes in culture. Nature, 274:813-816.

Raine, C.S. (1984) Morphology of myelin and myelination. In: Myelin. P. Morell, ed. Plenum Press, New York, pp. 1-50.

Rivier, J., Galyean, R., Gray, W.R., Azimi-Zonooz, A., McIntosh, J.M., Cruz, L.J., and Olivera, B.M. (1987) Neuronal calcium channel in- 
hibitors. Synthesis of omega-conotoxin GVIA and effects on $45 \mathrm{Ca}$ uptake by synaptosomes. J. Biol. Chem., 262:1194-1198.

Schmidt, M.F., Bandtlow, C.E., Hassinger, T.D., Schwab, M.E., and Kater, S.B. (1991) CNS myelin growth inhibitor NI-35 causes a large transient rise in intracellular calcium which precedes growth arrest and collapse of rat DRG growth cones. Neurosci. Abstr., 17:927.

Verkhratsky, A.N., Trotter, J., and Kettenmann, H. (1990) Cultured glial precursor cells from mouse cortex express two types of calcium currents. Neurosci. Lett., 112:194-198.
Wood, P. and Bunge, R.P. (1984) The tiology of the oligodendrocyte. In: Oligodendroglia; Advances in Neurochemistry, Vol. 5. W.T. Norton, ed. Plenum Press, New York, pp. 1-40.

Yaari, Y., Hamon, B., and Lux, H.D. (1987) Development of two types of calcium channels in cultured mammalian hippocampal neurons. Science, 235:680-682. 